\title{
Modal Analysis of Beam Oscillation
}

\author{
Barbara Schürger ${ }^{1, *}$, Michal Kicko ${ }^{1}$, Vojtech Neumann ${ }^{1}$, Peter Frankovský ${ }^{1}$ \\ Technical university of Košice, Faculty of Mechanical Engineering, Department of Applied Mechanics and Mechanical Engineering, Letná 9, 04200
} Košice, Slovak Republic

\begin{abstract}
This paper deals with modal analysis through 3 different approaches, namely: analytically, numerically and experimentally. The investigated mechanical structure in this case will be a beam, which is characterized by three parameters, one of which is significantly larger than the other two. The beam is a structural element designed to transmit forces, mostly external ones. Modal analysis of the beam will be performed in 2 versions of its fixation, as a free structure and a one-sided embedded fixation. Modal parameters obtained by 3 different approaches will be compared with each other, which will determine whether the method of calculation was chosen correctly or whether the experiment was performed correctly.
\end{abstract}

Keywords: modal analysis; beam; modal parameters.

\section{Introduction to modal analysis}

Modal analysis as a scientific discipline belongs to the highly extensive methods. We use it to describe the dynamic behaviour of mechanical structures by decomposing a complex oscillating action (Figure 1) into partial actions, which are characterized by modal parameters, namely natural frequency, natural shape of oscillations and damping. Thanks to these parameters, we can assume the properties and dynamic behaviour of the mechanical structure [1-3].

We can use modal analysis in 2 different levels, in the theoretical and practical level. In the theoretical level as a calculation method and in the practical level by direct implementation of experimental measurement of a specific mechanical structure, the so-called modal test [1-3].

In technical practice, the comparison of the results of modal parameters obtained by the FEM method or another theoretical method with the results obtained experimentally is used [4].

The natural frequencies obtained by the modal analysis help us to determine the critical operating states that must not occur in the mechanical system. This is a dangerous question of the resonance of the system, which arises when the natural frequencies coincide with the frequencies of the excitation forces. The principle is therefore to combine two oscillations with the same frequencies, resulting in a resonant frequency (Figure 2). With this frequency match, even small excitation forces elicit a high response. Resonance in the mechanical system causes increased noise and significant vibration, resulting in reduced life and damage to the system [5-7].

A simple case of resonance can be seen in this graph. Frequency $f_{B}$ indicates the resonant frequency. It is clear from the figure that at a lower frequency $f_{A}$, but also at a higher frequency $f_{C}$, the amplitude is significantly lower than at the resonant frequency $f_{B}$, where the amplitude we do not even see [5-7].

The proper shapes of the oscillations obtained by modal analysis help us also to determine the places of maximum deformation. Thanks to this, it is possible to implement several system optimizations, such as geometric optimization, material optimization, 


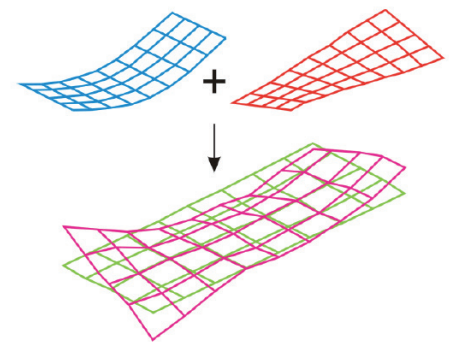

Figure 1: Modal decomposition

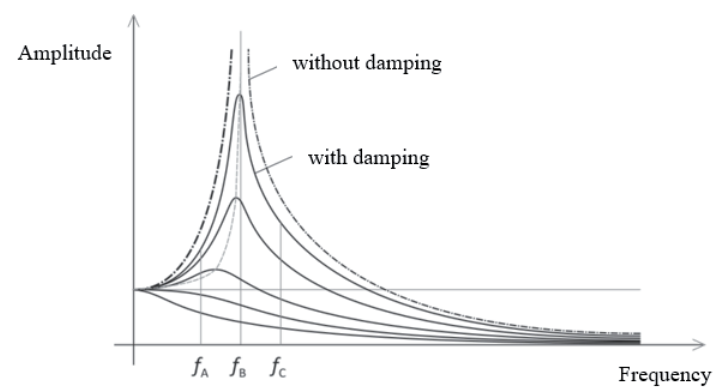

Figure 2: A simple case of resonance.

shape optimization or addition of supporting parts, etc. The purpose of this optimization is to eliminate dangerous oscillations [8].

According to the authors [9-11], Figure 3 shows how the optimization cycle proceeds through the individual phases from the input, which is our CAD model of the part, to the output, which represents the final shape of the part after optimization, not only in modal analysis, but also in general.

In technical practice, the comparison of the results of modal parameters obtained by the FEM method or another theoretical method with the results obtained experimentally is then used. There are several reasons for using both areas [12-13]:

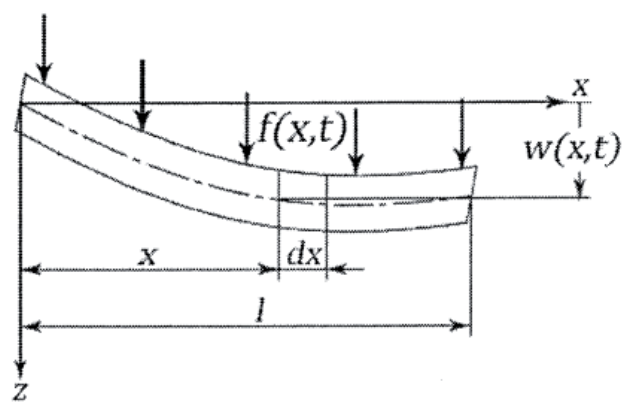

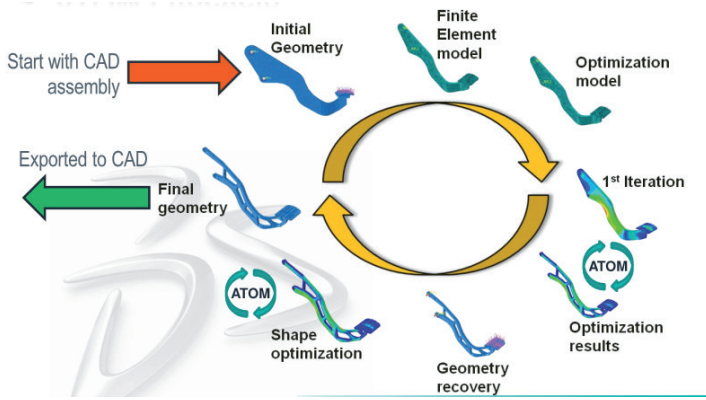

Figure 3: Optimization cycle

- correction of the theoretical model in such a way that it better corresponds to the actual measured values,

- verification of theoretical results with experimentally obtained data before further calculations,

- correlation of theoretical and experimental data to determine discrepancies between the two sets

\section{Bending stress analysis on beam}

First, it is necessary to define several valid conditions and relationships, which were defined in publication by the authors [8]:

- Moment of inertia and shear deformation are neglected.

- The cross section of beam $A(x)$ is constant along its entire length.

- $E J(x)$ is constant and the beam is symmetrical along the neutral axis.

- No axial forces are present in the longitudinal direction.

The considered beam is bend in the transverse direction with a length 1 and a rectangular crosssection $A(x)$ as shown in Figure 4. The flexural stiffness of the beam is $E J(x)$, where $E$ is the Young's modulus and $\mathrm{J}(x)$ is the quadratic moment of the cross section around the $y$-axis [8].

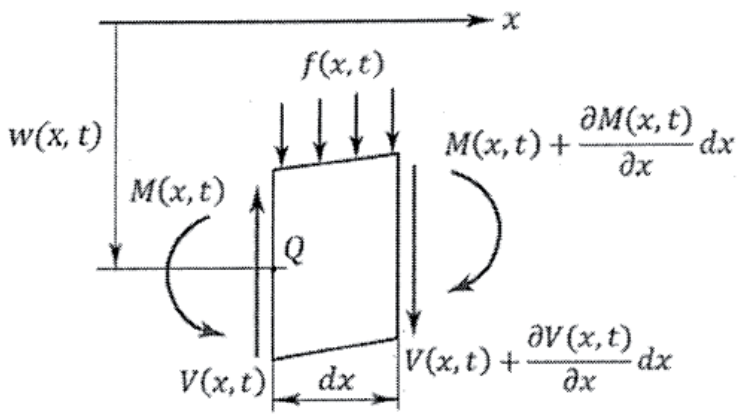

Figure 4: Scheme of considered beam bending stress. 
From the Euler-Bernoulli theory of beams, the relation for the bending moment $M(x, t)$ and the deflection in the direction of the z-axis $w(x, t)$ is applied [8]:

$$
M(x, t)=E J(x) . \partial w(x, t) / \partial x^{2}
$$

By successive modifications of Equation 1, we finally obtain a relation for the analytical calculation of natural frequencies in the form [8]:

$$
\omega=\beta^{2}(E J / \rho A)^{1 / 2}
$$

where $\beta$ is a coefficient determined from the boundary conditions of the equation and $\rho$ is bulk density or density of the material.

\section{Analytical calculation}

For the analytical calculation to be performed correctly, it is necessary to consider part geometry, material type (isotropic, anisotropic), variable plate thickness, applied loads (forces, moments), boundary conditions (F - "FREE", C - " CLAMPED ", SS -" SIMPLY SUPPORTED ").

In our case, the following characteristics were used:

- $\rho=7832 \mathrm{~kg} / \mathrm{m}^{3}$

$-I=400 \mathrm{~mm}$

$-h=40 \mathrm{~mm}$

$-b=8 \mathrm{~mm}$

$-E=210 \mathrm{GPa}$

$-\mu=0,3$

We write the boundary conditions for our type of problem as follows: F-F-F-F for free boundary condition and C-F-F-F for one-sided embedded fixation.

Based on the knowledge of the relations from the previous chapter, it is possible to calculate the natural frequencies of the mechanical structure analytically using the relation (2). However, it is still necessary to define the values of the coefficient $\beta$. The values of the coefficient $\beta$, similarly to the integration constants, are determined from the boundary conditions of the beam. Table 1 lists the first four values of $\beta \mathrm{n}$ for the basic cases of beam mounting loosely at both ends and with one end free and the other inverted [14].

By successively substituting the values of geometric characteristics and the values of the coefficient $\beta n$ into equation (2), the first four natural frequencies for a loosely placed and unilaterally embedded beam were analytically calculated. The results are shown in Table 2.

Table 1: Values of the coefficient $\beta n$

\begin{tabular}{|l|l|l|} 
& $\beta n$ for freestructure & $\beta n$ for one-sided embedded fixation \\
\hline$\beta 1$ & 4,730041 & 1,875104 \\
\hline$\beta 2$ & 7,853205 & 4,694091 \\
\hline$\beta 3$ & 10,995608 & 7,854757 \\
\hline$\beta 4$ & 14,137165 & 10,995541 \\
\hline
\end{tabular}

Table 2: Results of natural frequency values for both types of boundary condition using analytical calculation.

\begin{tabular}{|l|l|l|}
\hline Natural frequency $(\boldsymbol{H z})$ & Freestructure & \multicolumn{1}{|c|}{ One-sided embedded fixation } \\
\hline$\omega 1$ & 267,5479 & 42,0458 \\
\hline$\omega 2$ & 737,50622 & 263,4973 \\
\hline$\omega 3$ & 1445,8098 & 737,7992 \\
\hline$\omega 4$ & 2389,9968 & 1445,7922 \\
\hline
\end{tabular}

\section{Experimental measurement}

The measurement procedure in the experimental part of the modal analysis can be divided into the following stages [14]:

1. Pre-preparation of experimental measurement

a) Creation of a network of measuring points directly on the analysed component

b) Determination of excitation and measurement point.

c) Appropriately chosen placement of the measured structure, according to the specified boundary conditions.

d) Selection of excitation method (dynamic exciter or modal hammer).

e) Selection of force and response sensors (acceleration, velocity and deflection). It is often advisable to choose contactless sensors.

f) Analyzer configuration for signal processing.

g) Calibration of sensors and checking the settings of the measuring technique.

2. Measurement of modal data

a) Application of the response sensor (or adjustment of the laser vibrometer beam) to the measuring point.

b) Excitation of the structure by the selected type of exciter.

c) Recording of sensed signals at measuring / excitation points.

3. Analysis of measured data

a) Evaluation of measured results. Subsequent comparison with values obtained through calculation procedures (analytical / numerical).

b) Possibility to extend the experiment by harmonic analysis, while creating an amplitude-frequency characteristic.

The first step of the measurement was to assemble the measuring apparatus. The measurement was performed on two beams, which were identical in their geometric characteristics, only 
the method of their fixation was different. It is for this reason that the whole measurement procedure had to be repeated twice [15-25].

The specific method of their fastening is shown in Figure 5, namely:

1. In the first case, the beam is placed as F-F-F-F for free boundary condition (right arrow)

2. In the second case, the beam is placed as C-F-F-F for one-sided embedded fixation. (left arrow)

(a)

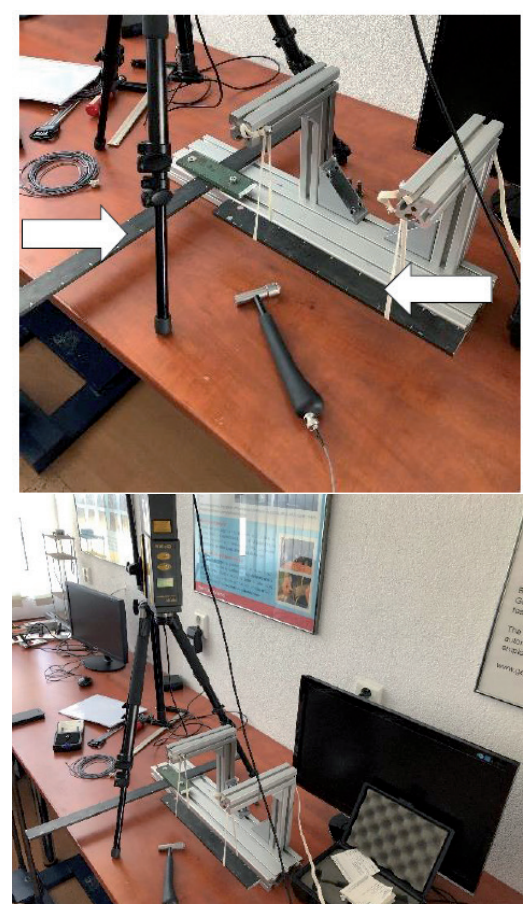

Figure 5: (a) Types of fixation of the measured beam. (b) Measurement system configuration.

A network of measuring points was then defined on the beams (1.). Excitation using a modal hammer (2.) was chosen as the excitation method. In the next step, a reflective element was applied to the beam, to which a laser beam generated by a Polytec PDV 100 (3.) vibrometer was directed. With the help of the reflective element, it is possible to monitor the magnitude of the deflection.

We connected the measuring system according to the instructions, checked the settings of the measuring technology given by the manufacturer and calibrated the sensor. Subsequently, according to Figure 6, the entire measuring system was configured in the Pulse software.

After connecting the measuring system, we created a geometric model in the Pulse software corresponding to the real system. On the created model, we defined the points at which the beam was excited by a modal hammer. Subsequently, we determined the point at which the response will be measured via the coordinates $[0.4 ; 3.75]$ and we defined the degrees of freedom. The $z^{+}$direction (point 19) is the measurement direction and the $z$ (points 1-18) is the excitation direction.

(a)

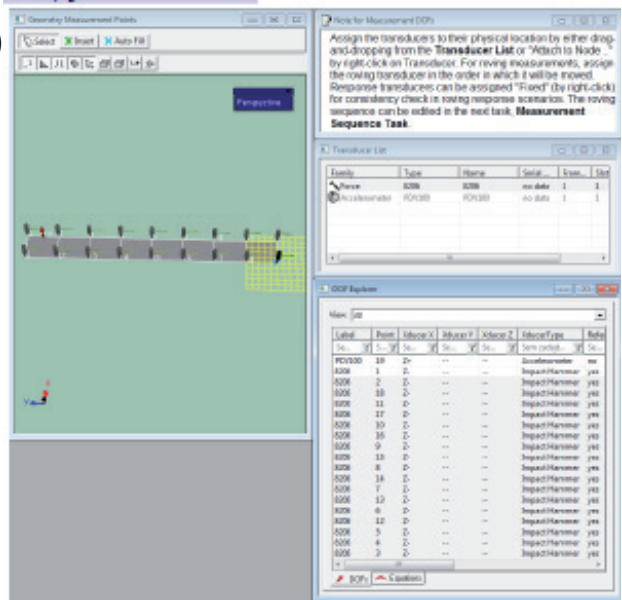

(b)

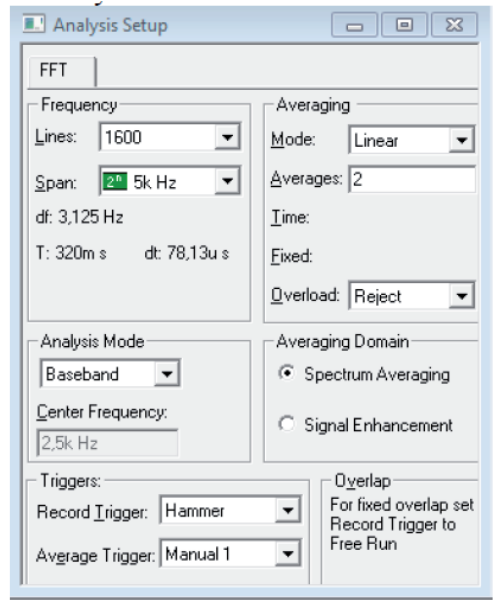

(c)

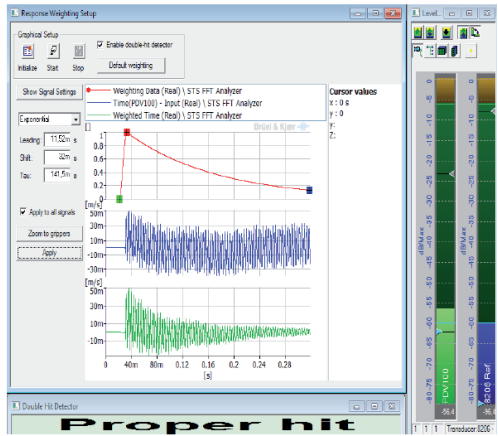

Figure 6: (a) Configuration of the system. (b) Measurement range. (c) Program weighing 
Table 3: Results of experimental modal analysis

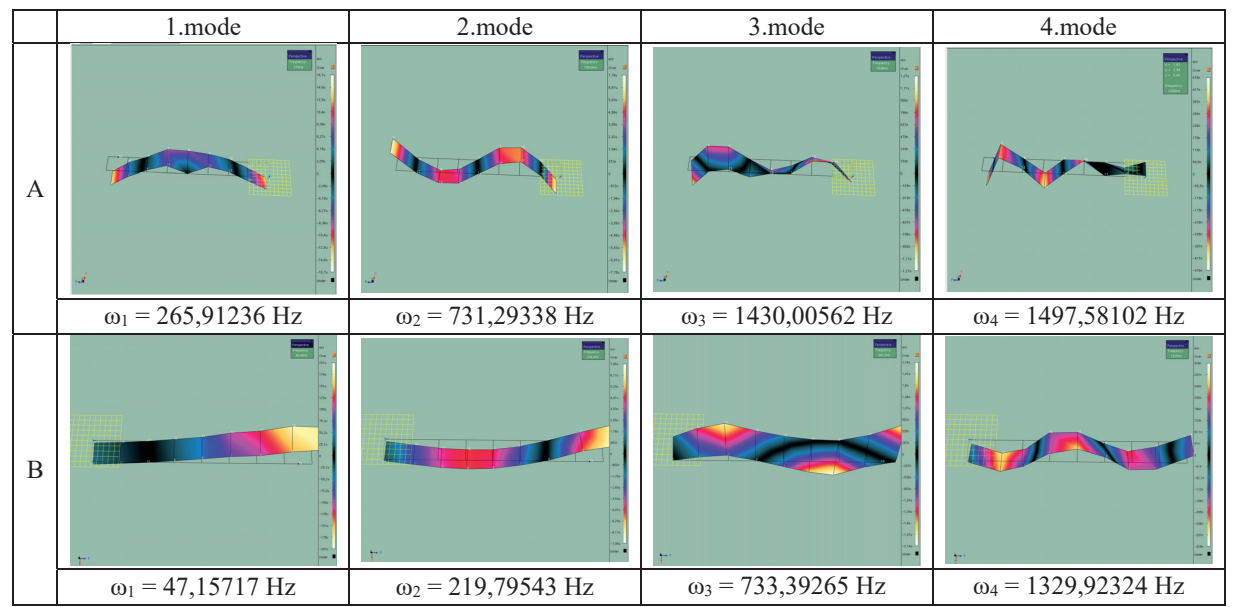

Table 4: Results of numerical modal analysis



Using a modal hammer, we brought the excitation to predefined points, while obtaining the response of such a system. By analysing the experimentally measured data, we obtained the modal parameters of the system, which in our case represented the natural frequencies and their own natural oscillation shapes.

The following figures in Table 3 show the first four mode shapes with the respective natural frequencies for a free boundary condition (variant A) and for a one-sided embedded fixation (variant B).

\section{Numerical analysis}

The finite element method in the NX Nastran calculation program was used in the numerical analysis. First, a model of the beam with the specified dimensions and characteristics from Chapter 3 was created as CAD model. The model created in this way was then covered by a finite element mesh with an element size of $4 \mathrm{~mm}$ (Figure 7).

In the next step, the numerical calculation of modal properties called frequency analysis was

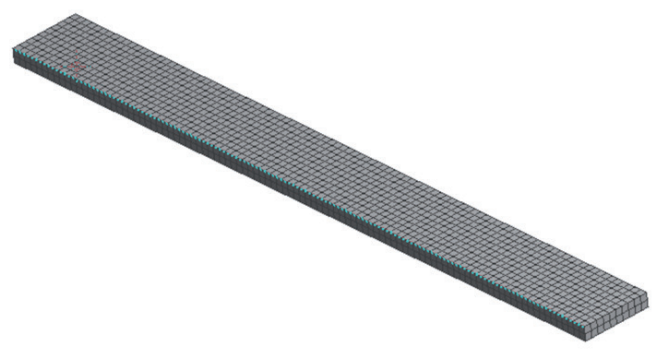

Figure 7: Finite element mesh on a modelled beam.

started (in NX Nastran, this solver is referred to as SOL 103 Real Eigenvalues). The values of natural frequencies were obtained by numerical calculation in the form of a simple table. We then transferred the values obtained in this way to the calculation tree and started the calculation again. With this step, we also obtained a graphical display and animation of the beam's own vibration shapes.

The following figures in Table 4 show the first four mode shapes with the respective natural frequencies for a free boundary condition (variant A) 
and for a one-sided embedded fixation (variant B).

\section{Conclusions}

In this paper, a modal analysis of the beam as one of the most used structural elements was performed. For two types of fixation - free boundary condition and one-sided embedded fixation modal parameters were experimentally obtained in the form of a graphical representation of the natural shapes of the beam oscillations and their corresponding values of natural frequencies.

To verify the experimentally measured data, a numerical analysis was performed in the NX program, where the proper shapes and frequencies of the beam oscillation were again obtained. At the same time, these data were supplemented by an analytical calculation of natural frequencies, which was performed based on derived relationships and available experimentally determined constants for both types of storage.

The summary results of the analytical, experimental and numerical calculation are given in Table 5 for a free boundary condition (variant A) and for a one-sided embedded fixation (variant B).

Finally, all data were compared, and it can be stated that the differences between the individual values are generally greater with one-sided entry of the beam

The reason for this difference was the impossibility of realizing such a perfect tight coupling in experimental measurements, with which the software works in numerical calculation. As a result, the natural frequency values obtained experimentally and numerically differ at higher natural frequencies, but the difference is always within the error limits caused by the measurement. Therefore, we can say that the performed measurement was correct.

\section{Acknowledgments}

The paper was created as part of the projects KEGA 027TUKE4/2020 and VEGA 1/0290/18.

Table 5: Results of three types of modal analysis

\section{References and Notes}

1. Bilošová, A (2012). Applied mechanic as part of a team of designers and developers (in Czech). Technical university of Ostrava, Ostrava.

2. Bocko, J, Segl'a, Š, Huňady, R (2016). Vibration of mechanical systems (in Slovak). Faculty of Mechanical Engineering, Technical University of Košice, Košice.

3. Delyová, I, Frankovský, P, Bocko, J, Trebuňa, P, Živčák, J, Schürger, B, Janigová, S (2021). Sizing and Topology Optimization of Trusses Using Genetic Algorithm. Materials, vol.14, no.4, pp. 715.

4. Kirthana, S, Nizamudin, N (2018). Finite Element Analysis and Topology Optimization of Engine Mounting Bracket. Mechanical Structures, vol. 13, no. 4, pp. 123-125.

5. Krishna, G, Tarak, D, Burman, R (2019). Numerical and experimental comparative study of Aluminium and hybrid mounting interfaces of launch vehicle avionics for weight reduction. Kanchan Bagh, Hyderabad.

6. Liang, L (2021). A user-defined element for dynamic analysis of saturated porous media in ABAQUS. Computers and Geotechnics, vol. 5, no. 8, pp. 49-54.

7. Li, Z, Zhang, C (2021). Design and parameter optimization of contactless vertical inductive angle sensor. Vacuum, vol. 169, no.23, pp. 141-156.

8. Vo, D, Borkovic, A (2020). Dynamic multi-patch isogeometric analysis of planar Euler-Bernoulli beams. Computer Methods in Applied Mechanics and Engineering, vol. 372, no.3, pp. 41-46.

9. Dong, X, Lian, J (2018). Structural vibration monitoring and operational modal analysis of offshore wind turbine structure. Mechanical Structures, vol.30, no.5, pp. 280-297.

10. Klarbring, A, Christensen, P (2009). An Introduction to Structural Optimization. Solid Mechanics and its Applications. Springer Science + Business Media B.V., Amsterdam.

11. Li, J, Guo, H (2016). Power generation quality analysis and geometric optimization for solar chimney power plants. Mechanical Structures, vol.35, no.15, pp. 228-237.

12. Olason, A, Tidman, D (2010). Methodology for Topology and Shape Optimization in the Design Process. Chalmers University of Technology, Division of Dynamics, Göteborg.

13. Parkinson, A, Balling, R (2013). Optimization Methods for

\begin{tabular}{|l|l|l|l|l|l|l|}
\hline \multicolumn{4}{|l|}{ Variant A } & \multicolumn{2}{l|}{ Variant B } \\
\hline Natural frequency $(\mathrm{Hz})$ & Analytically & Experimentally & Numerically & Analytically & Experimentally & Numerically \\
\hline$\omega 1$ & 267,5479 & 265,91236 & 263,692 & 42,0458 & 47,15717 & 41,708 \\
\hline$\omega 2$ & 737,50622 & 731,29338 & 725,561 & 263,4973 & 219,79543 & 260,82 \\
\hline$\omega 3$ & 1445,8098 & 1430,00562 & 1419,148 & 737,7992 & 733,39265 & 728,675 \\
\hline$\omega 4$ & 2389,9968 & 2355,56978 & 2331,261 & 1445,7922 & 1329,92324 & 1423,995 \\
\hline
\end{tabular}


Engineering Design. Brigham Young University, Brigham.

14. Wójcicki, Z, Grosel, J (2015). Experimental (OMA) and Numerical (FEM) Modal Analysis of Ball Mill Foundations. Accident Analysis \& Prevention, vol. 111, no. 4, pp. 858-863.

15. Song, B, Wang, H (2020). Dynamic simulation and optimization of clamping mechanism of online tension testing machine for wire ropes. Engineering Failure Analysis, vol. 95, no. 5, pp. 181-190.

16. Bathe, K (1982). Finite Element Procedures in Engineering Analysis. Inc. Englewood Cliffs, New Jersey: Prentice Hall.

17. Skaar, K (2013). Parameterization and Multiobjective Optimization. Norwegian University of Science and Technology, Trondheim.

18. Bendsoe, M, Sigmund, O (2003). Topology Optimization. Theory, Methods and Applications. Springer, New York.

19. Olivieri, C, De Paulis, F, Orlandi, A, Pisani, C, Giannuzzi, G, Salvati, R, Zaottini, R (2020). Estimation of Modal Parameters for Inter-Area Oscillations Analysis by a Machine Learning Approach with Offline Training. Energies, vol. 13, no. 23, pp. 640-645.

20. Musiał, M, Trapko, T, Grosel, J (2021). Static and Dynamic Stiffness of Reinforced Concrete Beams Strengthened with Externally Bonded CFRP Strips. Materials, vol. 14, no. 4, pp. 910-917.

21. Kalybek, M, Bocian, M, Nikitas, N (2021). Performance of Optical Structural Vibration Monitoring Systems in Experimental Modal Analysis. Sensors, vol. 21, no. 4, pp. 1239-1245.

22. Le, V, Goo, N (2020). Dynamic Characteristics and Damage Detection of a Metallic Thermal Protection System Panel Using a Three-Dimensional Point Tracking Method and a Modal Assurance Criterion. Sensors, vol. 20, no. 24, pp. 718726.

23. Zimmaro, P, Ausilio, E (2020). Numerical Evaluation of Natural Periods and Mode Shapes of Earth Dams for Probabilistic Seismic Hazard Analysis Applications. Geosciences, vol. 10, no. 12, pp. 499-508.

24. Drliciak, M, Celko, J, Cingel, M, Jandacka, D (2020). Traffic Volumes as a Modal Split Parameter. Sustainability, vol. 12, no. 24, pp. 49-54.

25. Sivák, P, Frankovský, P, Delyová, I, Bocko, J, Kostka, J, Schürger, B (2020). Influence of Different Strain Hardening Models on the Behavior of Materials in the Elastic-Plastic Regime under Cyclic Loading. Materials, vol. 13, no. 23, pp. 53235330.

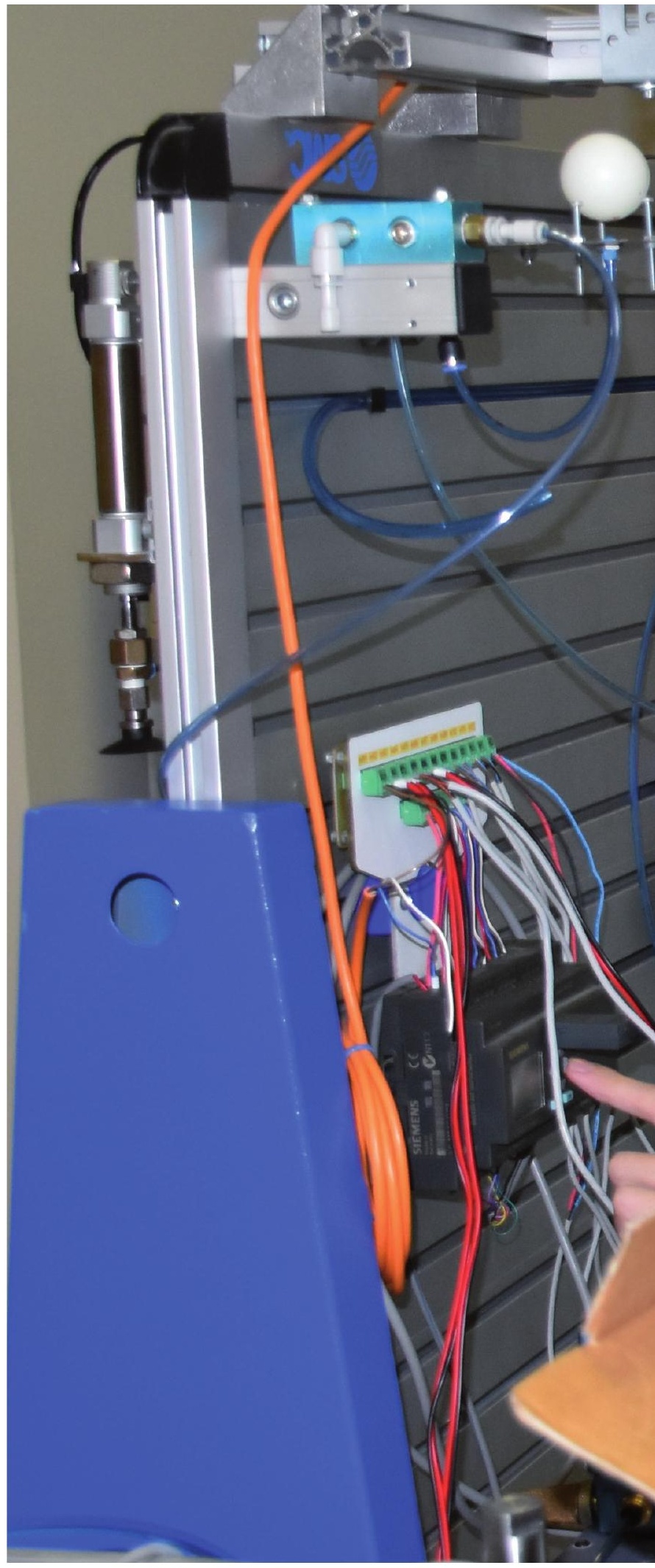

